BMJ

Open

Gastroenterology

\title{
High incidence of glucocorticoid- induced hyperglycaemia in inflammatory bowel disease: metabolic and clinical predictors identified by machine learning
}

\author{
Martin McDonnell (D , ,,2 Richard J Harris, ${ }^{1}$ Florina Borca, ${ }^{3,4}$ Tilly Mills, ${ }^{1}$ \\ Louise Downey, ${ }^{1}$ Suranga Dharmasiri, ${ }^{1}$ Mayank Patel, ${ }^{5}$ Benjamin Zare, \\ Matt Stammers, ${ }^{1,6}$ Trevor R Smith, ${ }^{1}$ Richard Felwick, ${ }^{1} \mathrm{~J}$ R Fraser Cummings (D) ,1,2 \\ Hang T T Phan, ${ }^{3,4}$ Markus Gwiggner ${ }^{1}$
}

To cite: McDonnell M, Harris RJ, Borca F, et al. High incidence of glucocorticoidinduced hyperglycaemia in inflammatory bowel disease: metabolic and clinical predictors identified by machine learning. BMJ Open Gastro 2020;7:e000532. doi:10.1136/ bmjgast-2020-000532

- Additional material is published online only. To view, please visit the journal online (http://dx.doi.org/10.1136/ bmjgast-2020-000532).

MM and RJH are joint first authors.

Received 31 August 2020 Revised 3 October 2020 Accepted 23 October 2020

Check for updates

(C) Author(s) (or their employer(s)) 2020. Re-use permitted under CC BY-NC. No commercial re-use. See rights and permissions. Published by BMJ.

For numbered affiliations see end of article.

Correspondence to Dr Martin McDonnell; m.j.mcdonnell@soton.ac.uk

\section{ABSTRACT}

Background Glucocorticosteroids (GC) are longestablished, widely used agents for induction of remission in inflammatory bowel disease (IBD). Hyperglycaemia is a known complication of GC treatment with implications for morbidity and mortality. Published data on prevalence and risk factors for GC-induced hyperglycaemia in the IBD population are limited. We prospectively characterise this complication in our cohort, employing machine-learning methods to identify key predictors of risk.

Methods We conducted a prospective observational study of IBD patients receiving intravenous hydrocortisone (IVH). Electronically triggered three times daily capillary blood glucose (CBG) monitoring was recorded alongside diabetes mellitus (DM) history, IBD biomarkers, nutritional and IBD clinical activity scores. Hyperglycaemia was defined as $C B G \geq 11.1 \mathrm{mmol} / \mathrm{L}$ and undiagnosed $\mathrm{DM}$ as glycated haemoglobin $\geq 48 \mathrm{mmol} / \mathrm{mol}$. Random forest (RF) regression models were used to extract predictor-patterns present within the dataset.

Results 94 consecutive IBD patients treated with IVH were included. $60 \%$ (56/94) of the cohort recorded an episode of hyperglycaemia, including $57 \%$ (50/88) of those with no history of DM, of which 19\% (17/88) and $5 \%$ (4/88) recorded a CBG $\geq 14 \mathrm{mmol} / \mathrm{L}$ and $\geq 20 \mathrm{mmol} / \mathrm{L}$, respectively. The RF models identified increased $\mathrm{C}$-reactive protein (CRP) followed by a longer IBD duration as leading risk predictors for significant hyperglycaemia.

Conclusion Hyperglycaemia is common in IBD patients treated with intravenous GC. Therefore, CBG monitoring should be included in routine clinical practice. Machine learning methods can identify key risk factors for clinical complications. Steroid-sparing treatment strategies may be considered for those IBD patients with higher admission CRP and greater disease duration, who appear to be at the greatest risk of hyperglycaemia.

\section{INTRODUCTION}

In the 1950s Truelove and Witts performed the first randomised controlled trial of
Summary box

What is already known about this subject?

- Glucocorticosteroids (GC) are long-established induction agents in the management of inflammatory bowel disease (IBD) and prescribing remains widespread.

- Hyperglycaemia is a known complication of GC and has been linked to morbidity and mortality. The frequency and risk factors for this complication in the inpatient IBD population is not known.

What are the new findings?

- $57 \%$ of IBD patients treated with intravenous hydrocortisone (IVH), developed a hyperglycaemia (capillary blood glucose (CBG) $\geq 11.1 \mathrm{mmol} / \mathrm{L}$ ) during admission.

- C-reactive protein (CRP) and IBD duration are the strongest predictors of $\mathrm{CBG}$ elevation.

How might it impact on clinical practice in the foreseeable future?

- Monitoring and management of blood glucose should be routine practice for all IVH-treated hospitalised IBD patients, particularly those with a high inflammatory burden.

- There is an emergent case for research into steroidfree management of acute IBD.

glucocorticosteroids (GC) in active ulcerative colitis (UC), demonstrating a reduction in mortality from $10.9 \%$ to $4.6 \%$ after 2 months of treatment. ${ }^{1}$ Seventy years later, GC remains a common treatment for inflammatory bowel disease (IBD); a recent UK multicentre study identified $30 \%$ of patients were exposed to GC in the preceding 12 months. ${ }^{2}$ GC are firstline induction agents for moderate to severe Crohn's disease (CD) and UC in consensus 
guidelines and intravenous GC are widely used as the initial medical therapy for IBD patients admitted to hospital with severe disease flares. ${ }^{34}$

The mechanism of action of GC is mediated via the cytosolic glucocorticoid receptor (cGR). After GC binding, cGR-associated proteins dissociate, and the GC-cGR complex translocates to the nucleus. The nuclear GC-cGR complex binds to DNA, upregulating the transcription of anti-inflammatory cytokines (eg, interleukin (IL)-10, annexin 1) and downregulating transcription of proinflammatory mediators (eg, IL-1 $\alpha$, IL-1 $\beta$, IL-8, TNF $\alpha$ ). More rapid, non-genomic, effects occur via both the cGR dissociated proteins and likely via other novel transmembrane receptors. ${ }^{5}$ Steroid-induced hyperglycaemia is mediated by decreased $\beta$-cell insulin production, increased insulin resistance and increased gluconeogenesis. ${ }^{6}$

Studies in other GC-treated populations demonstrate both short-term and long-term severe adverse effects including ocular, musculoskeletal, cardiovascular, metabolic and infectious complications. ${ }^{7}$ In addition, de-novo hyperglycaemia and deterioration of glycaemic control in those with pre-existing diabetes mellitus (DM) have been recognised adverse effects of GC since the 1950s. ${ }^{8}$ The specific risk in IBD hospitalised patients with severe IBD flares, however, is not known.

Hyperglycaemia has been associated with increased length of stay, morbidity and mortality in non-critical care medical and surgical patients. ${ }^{9}$ At least 67 cases of GC induced metabolic emergencies including ketoacidosis

Table 1 Demographics

\begin{tabular}{|c|c|c|c|c|}
\hline & & CD & IBDU & UC \\
\hline & $\mathbf{n}$ & 54 & 4 & 36 \\
\hline Age & & $42.2(37.9-46.5)$ & $49.8(28.4-71.1)$ & $43.1(37.0-49.2)$ \\
\hline Gender & Female & $25(46.3)$ & $2(50.0)$ & $19(52.8)$ \\
\hline $\mathrm{BMI}$ & $<18.5$ & $6(11.1)$ & $1(25.0)$ & $3(8.3)$ \\
\hline & $18.5-25$ & $28(51.9)$ & $2(50)$ & $16(44.4)$ \\
\hline & $25-30$ & $13(24.1)$ & $1(25)$ & 14 (38.9) \\
\hline & $>30$ & $7(13.0)$ & $0(0.0)$ & $3(8.3)$ \\
\hline MUST score & 0 & $17(31.5)$ & $1(25.0)$ & $17(48.6)$ \\
\hline & 1 & $13(24.1)$ & $1(25.0)$ & $10(28.6)$ \\
\hline & 2 & $13(24.1)$ & $2(50.0)$ & $4(11.4)$ \\
\hline & $\geq 3$ & $11(20.4)$ & $0(0.0)$ & $4(11.4)$ \\
\hline Length of diagnosis (years) & & $7.3(4.6-10.0)$ & $3.3(0-8.4)$ & $5.0(3.2-6.8)$ \\
\hline History of diabetes & No & $49(90.7)$ & $4(100.0)$ & $35(97.2)$ \\
\hline & Type 2 DM & $5(9.3)$ & $0(0.0)$ & $1(2.8)$ \\
\hline Admission $\mathrm{HbA} 1 \mathrm{c}$ & & $38.5(35.5-41.5)$ & $37.0(33.1-40.8)$ & $37.7(35.8-39.6)$ \\
\hline Admission calprotectin & & 2854 (1980-2298) & 2491 (NA) & 3159 (2302-4071) \\
\hline Admission CRP & & $75.9(50.5-101.3)$ & $138.0(106.7-169.3)$ & $88.9(53.6-124.1)$ \\
\hline PMS & & - & $5.50(4.5-6.5)$ & $7.78(7.2-8.3)$ \\
\hline $\mathrm{HBI}$ & & $14.7(13.0-16.5)$ & - & - \\
\hline Current treatment & Oral steroids & $8(14.8)$ & $1(25)$ & $6(16.7)$ \\
\hline & Oral 5ASA & $2(3.7)$ & $2(50)$ & $20(55.6)$ \\
\hline & Immunomodulator & $18(33.3)$ & $0(0)$ & $6(16.7)$ \\
\hline & Anti-TNF & $10(19.2)$ & $0(0)$ & $10(29.4)$ \\
\hline & Vedolizumab & $2(3.8)$ & $0(0)$ & $0(0.0)$ \\
\hline & Ustekinumab & $7(13.5)$ & $0(0)$ & $0(0.0)$ \\
\hline Smoking & Current & $12(23.5)$ & $0(0)$ & $0(0.0)$ \\
\hline & Ex-smoker & $9(17.7)$ & $1(25.0)$ & $13(36.1)$ \\
\hline & Never smoked & $30(58.8)$ & $3(75.0)$ & $23(63.9)$ \\
\hline
\end{tabular}

For categorical variables $\mathrm{n}(\%)$ shown, for continuous variables mean $(95 \% \mathrm{Cl})$ shown.'

anti-TNF, anti-tumour necrosis factor; 5-ASA, 5-aminosalicylic acid; BMI, body mass index; CRP, C-reactive protein; DM, diabetes mellitus; $\mathrm{HbA1c}$, glycated haemoglobin; HBI, Harvey Bradshaw Index; IBDU, inflammatory bowel disease-as yet unclassified; MUST, Malnutrition Universal Screening Tool; PMS, Partial Mayo Score. 
and hyperosmolar hyperglycaemic states have been reported, 11 of which were fatal. ${ }^{10}$ Following two cases of GC-related diabetic crisis at our institution in IBD patients with no prior diabetes, electronically triggered systematic capillary blood glucose (CBG) monitoring was introduced for all prescribed intravenous hydrocortisone (IVH). This allowed the systematic study of hyperglycaemia in hospitalised IBD patients receiving IVH.

\section{MATERIALS AND METHODS}

We conducted a prospective observational study of consecutive admissions of IBD patients treated with IVH between October 2017 and December 2018. Included subjects had a confirmed diagnosis of IBD (either historic or during admission episode) and received at least four doses of $100 \mathrm{mg}$ IVH over 24 hours (the standard dosing regimen for IBD flare at our centre).

Subjects had three times daily CBG monitoring automatically triggered by electronic prescription of IVH. CBG results were automatically digitally captured in the electronic patient record. Predictor variables included age, IBD diagnosis and subtype, IBD disease duration, admission IBD severity score (Harvey Bradshaw Index or Partial Mayo Score), serum biochemistry, glycated haemoglobin (HbA1c), faecal calprotectin, $\mathrm{C}$ reactive protein, potential DM risk factors (body mass index (BMI), family history, concomitant medications), self-reported weight loss and Malnutrition Universal Screening Tool score. ${ }^{11}$

Patients developing a $\mathrm{CBG} \geq 14 \mathrm{mmol} / \mathrm{L}$ were referred to the diabetes team for consideration of treatment. In an attempt to identify only those with de novo hyperglycaemia, subjects with a baseline HbA1c or CBG (fasting status unknown) above the threshold previously used to diagnose DM were counted as potentially undiagnosed DM $(\geq 48 \mathrm{mmol} / \mathrm{mol}$ and $\geq 11.1 \mathrm{mmol} / \mathrm{L}$, respectively). ${ }^{12}{ }^{13}$ Biochemical follow-up was performed at 1 year to assess repeat $\mathrm{HbA1c}$ measurements and serum and faecal biomarker assessments.

Laboratory values outside the quantification limit were substituted with the upper/lower limit value. Categorical and continuous predictors were handled and formatted appropriately. Data were analysed using Python V.3.7.4 and R V.3.6.1. Descriptive statistics were applied to cohorts, and Pearson correlations between independent and dependent variables were ascertained with matplotlib/seaborn heat-maps. Random forest (RF) machine learning models using the scikit-learn package V.0.22.1 were constructed to regress the phenotypic and admission clinical predictors. ${ }^{14} 15$ Pairwise correlations between input features (see online supplemental data) were identified to eliminate similar features. Then multivariable RF models were constructed and optimised using random grid search. Subsequently, fivefold crossvalidation with a train-test split was used to test effectiveness at predicting glucose levels in the internal holdout set. Mean squared error was the reported metric.

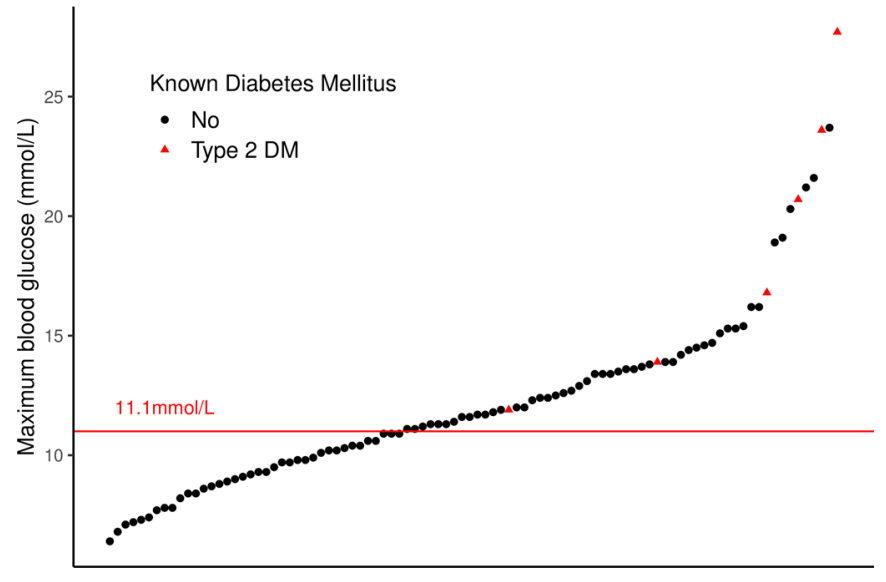

Figure 1 Maximum recorded capillary blood glucose for each admission plotted in ascending order. DM, diabetes mellitus.

\section{RESULTS}

\section{Demographics}

Ninety-four IBD inpatient episodes met the inclusion criteria; 54 for CD, 36 for UC and 4 for IBD-as yet unclassified (IBDU). The median length of IVH treatment was 4 days (range 1-11). Subject characteristic at time of hospital admission are described in table 1.

Five out of ninety-four episodes were for patients with a prior diagnosis of type $2 \mathrm{DM}$. One further subject had a pre-GC treatment CBG of $13.9 \mathrm{mmol} / \mathrm{L}$ and thus considered as potentially undiagnosed type $2 \mathrm{DM}$ and included in analyses as having prior DM.

\section{Incidence and management of hyperglycaemia}

The overall global incidence of hyperglycaemia in our cohort of hospitalised IBD patients receiving IVH was $60 \%$ (56/94), with intervention for diabetes initiated in $20 \%(11 / 56)$ of these patients (see figure 1$)$. For those without a prior diagnosis of DM, $57 \%$ (50/88) developed diabetic-range CBG $(\geq 11.1 \mathrm{mmol} / \mathrm{L})$ while $19 \%(17 / 88)$ and $5 \%(4 / 88)$ developed a $\mathrm{CBG} \geq 14 \mathrm{mmol} / \mathrm{L}$ and $\geq 20 \mathrm{mmol} / \mathrm{L}$, respectively. Twenty-five out of fifty of those with de-novo hyperglycaemia had $\mathrm{CD}, 22 / 50 \mathrm{UC}$, and $3 / 50$ IBDU with a combined mean age of 44.8 years (SD 17.4). In patients without a prior diagnosis of DM $14 \%$ $(7 / 50)$ had diabetic interventions; $6 / 50$ started an oral hypoglycaemic and $1 / 50$ commenced insulin therapy.

All six patients with prior DM (5CD and $1 \mathrm{UC}$ ) had an episode of hyperglycaemia and $4 / 6$ recorded a CBG $\geq 20 \mathrm{mmol} / \mathrm{L}$. Fifty per cent $(3 / 6)$ were started on insulin, and $17 \%(1 / 6)$ commenced oral hypoglycaemic therapy.

Of the 50 admissions in patients without prior DM recording a $\mathrm{CBG} \geq 11.1 \mathrm{mmol} / \mathrm{L}, 22 \%(11 / 50)$ were preceded directly by a course of oral steroids compared with $8 \%(3 / 38)$ without hyperglycaemia ( $\mathrm{p}=0.09$, Fisher's exact test). In $60 \%(30 / 50)$ peak CBG occurred on day 2 of IVH treatment, but in 14\% (7/50) of cases peak, CBG was not reached until 7 days after the first dose.

During index admission, 54\% (30/56) of hyperglycaemic patients and $50 \%(19 / 38)$ of normoglycaemic 


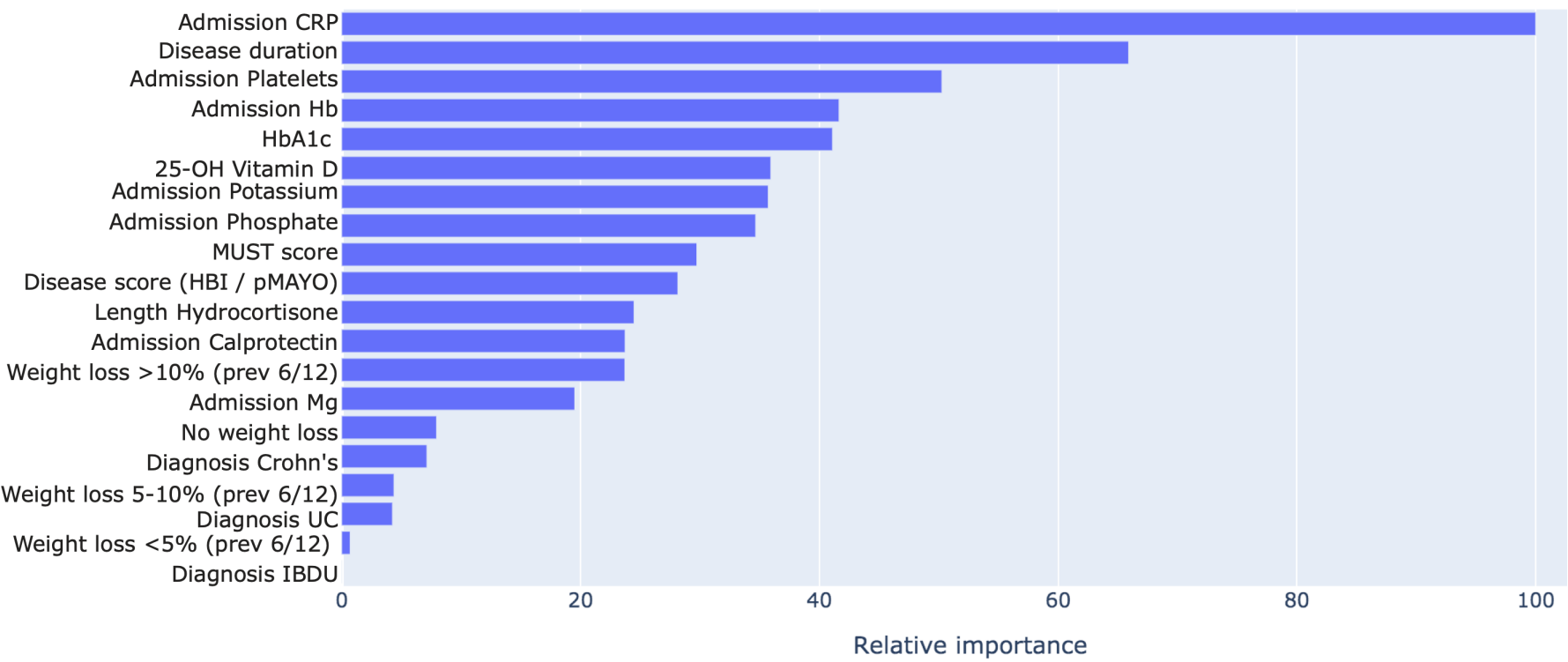

Figure 2 Relative variable importance for Random Forest modelling of maximum capillary blood glucose. CRP, C-reactive protein; HBI, Harvey Bradshaw Index; IBDU, inflammatory bowel disease-as yet unclassified; MUST, Malnutrition Universal Screening Tool; UC, ulcerative colitis.

patients were started on biological therapy. Four patients underwent emergency inpatient colectomy, all of whom had recorded a hyperglycaemic episode. Mean $( \pm 95 \%$ CI) length of stay was $12.6( \pm 3.0)$ days for hyperglycaemic patients and $9.1( \pm 2.0)$ days for normoglycaemic patients $(\mathrm{p}=0.053$, t-test $)$.

Physicians' decision making was influenced by steroidinduced hyperglycaemia, particularly in those with a persistent CBG $\geq 14 \mathrm{mmol} / \mathrm{L}$ (trigger for diabetes specialist review). Conventional post-IVH 8-week tapering course of oral prednisolone prescribing differed. Only $30 \%$ of patients with $\mathrm{CBG} \geq 14 \mathrm{mmol} / \mathrm{L}$ received oral prednisolone without intervention (eg, diabetic medication or rapid steroid taper) versus $58 \%$ of those with normoglycaemia.

\section{Predictors of hyperglycaemia}

Maximum CBG was positively correlated with CRP, platelet count and $\mathrm{HbAlc}$ and negatively correlated with haemoglobin (see correlation heat-map in online supplemental data). For non-diabetic patients, the RF model was fitted 500 times with a maximum depth of 90 trees using a random grid search method. CRP was the most critical predictor identified, followed by disease duration, platelet count, admission haemoglobin and HbA1c (see figure 2). The final mean squared error of the optimised non-diabetic patient model was 1.876 .

The variables contributing to the RF modelling and their respective importance of contribution are shown in figure 2. Age and BMI did not contribute to the model.

RF models optimised by grid search will rank the relative importance of each variable against the most critical variable (in this case, CRP). This provides a sense of how much each variable contributes to the predictive power of the overall model. In this instance, the length of IVH usage and metabolic variables are not as crucial to predicting hyperglycaemia as blood markers of inflammation.

Once the optimised RF model had been developed, blood glucose predictions were made on the holdout set using fivefold cross-validation to reduce overfitting.

Figure 3 reveals that the test-set predictions were comparable to the training set predictions. This pattern was present across all folds of the cross-validation; this graph is given only as an example of the model's performance.

When the RFR model included the six patients with $\mathrm{DM}$, the leading predictor of CBG elevation became

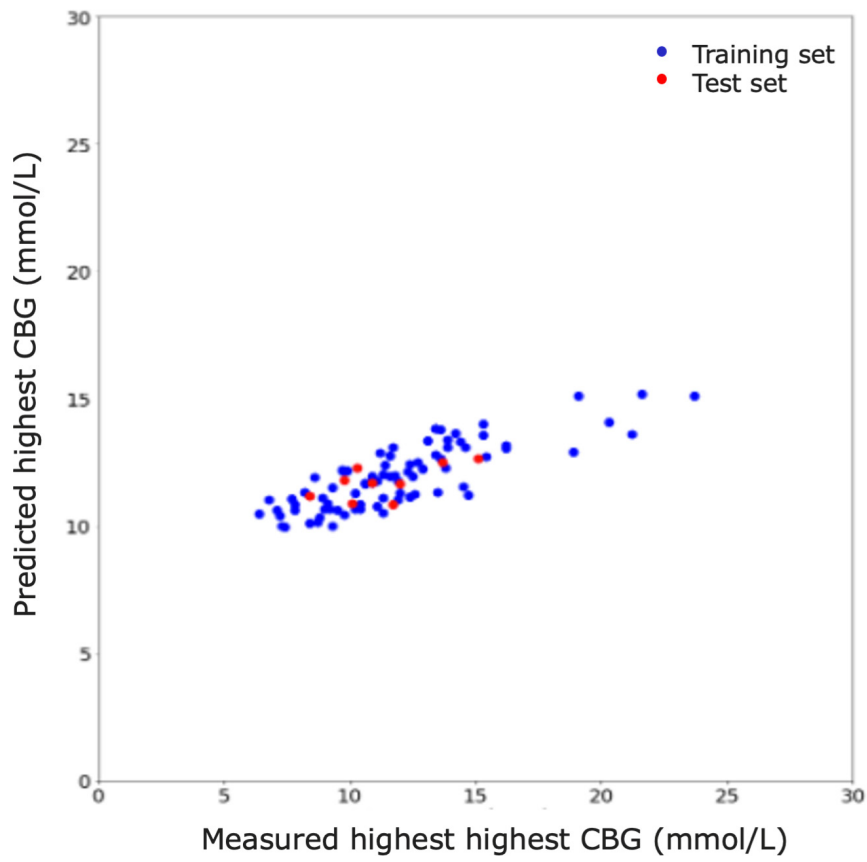

Figure 3 Predicted highest capillary blood glucose (CBG) vs measured highest CBG using model (subjects with history of Diabetes Mellitus excluded). CBG; capillary blood glucose. 
HbAlc (see online supplemental figure 3). Due to high CBG elevation $(\geq 20 \mathrm{mmol} / \mathrm{L})$ in subjects with known diabetes, particularly those with evidence of inadequate control by admission HbAlc, the RFR modelling shown above excluded these patients. Online supplemental data show RFR variable importance, correlation matrix and model with the patients with previous diabetes included. Their inclusion increased the mean squared error to 2.158 , which is not surprising given the differing preadmission characteristics in this subpopulation.

\section{Additional reported side effects and metabolic instability}

Thirty-two per cent $(30 / 94)$ of episodes included a patient-reported symptom of anxiety or mood change, $11 \%(10 / 94)$ of poor sleep and 10\% (9/94) a report of appetite change. Electrolyte depletion, like hyperglycaemia, is a marker of metabolic and nutritional dysregulation and was common in the whole cohort with no statistically significant differences between hyperglycaemic and normoglycaemic patients. Sixty-two per cent (58/94) recorded serum potassium below the lower limit of our laboratory reference range $(<3.5 \mathrm{mmol} / \mathrm{L})$, $71 \%(67 / 94)$ low serum magnesium $(<0.7 \mathrm{mmol} / \mathrm{L})$ and $84 \%$ (79/94) low serum phosphate $(<0.8 \mathrm{mmol} / \mathrm{L})$ (see online supplemental data).

The percentage weight loss in the preceding 6 months was calculated alongside BMI as part of malnutrition screening and triage of all patients as per standard UK hospital practice. ${ }^{16}$ Sixty-six per cent $(37 / 56)$ of hyperglycaemic patients and $57 \%$ (21/38) of normoglycaemic patients reported weight loss in the 6 months before admission, but neither BMI nor percentage weight loss category featured in the model to predict CBG. All patients were routinely assessed by a specialist dietitian who prescribed nutritional support as indicated; 53/94 had oral nutritional supplements, 5/94 nasogastric feeding and 5/94 parenteral nutrition. There was no significant difference in the proportion of hyperglycaemic versus normoglycaemic patients receiving nutritional therapy (see online supplemental data).

\section{One-year follow-up}

The follow-up clinical reviews and calprotectin postdischarge were part patient-triggered, and follow-up disease activity scoring was not universally available for our cohort. There was a significant decrease in faecal calprotectin over the 12-month follow-up. For those without hyperglycaemia during the acute episode, there was a trend to lower mean calprotectin at 12 months, 210 $( \pm 707)$ versus $738( \pm 640 \mathrm{p}=0.18 \mathrm{t}$-test $)$. In non-diabetic patients with raised calprotectin at admission and paired results $35 \%(6 / 17)$ normalised by 12 months in those who had hyperglycaemia and $75 \%(9 / 12)$ normalised in those without hyperglycaemia $\mathrm{p}=0.06$ (Fisher's exact test). There was no significant change in HbAlc over the 1-year follow-up for any groups.

\section{DISCUSSION}

Our data demonstrate that hyperglycaemia is common in IBD patients treated with IVH. By instituting electronically triggered regular CBG monitoring of IBD patients treated with $\mathrm{IVH}$, we have uncovered the high prevalence of this relevant complication. Our monitoring regime identified patients who required diabetic intervention or modification of their treatment in response to hyperglycaemia. The void of data regarding steroid-induced diabetes in IBD suggests that in current practice it may often be under-recognised, and thus under-treated, potentially exposing patients to the risk of complications such as increased length of stay and infection. Hyperglycaemia has also been mechanistically linked to intestinal barrier dysfunction in both murine models and non-IBD human subjects. ${ }^{17}$ Through this mechanism, it is at least theoretically possible to link hyperglycaemia directly to intestinal inflammation and IBD outcomes. ${ }^{18}$

Until now there has been no accurate prospective evidence of the extent and severity of IVH-induced hyperglycaemia in IBD. A previous retrospective study of steroid side effects in elderly CD patients estimated an incidence of just $17 \%$, but did not have systematic CBG monitoring. ${ }^{19}$ Likewise, studies of GC-treated rheumatology patients report the occurrence of hyperglycaemia at $9 \%-42 \% .^{20-22}$ A potential explanation for the higher frequency seen here is that our cohort only included hospitalised patients, who were both monitored frequently and likely to have had more significant systemic inflammation. The incidence we report is similar to that in a smaller study of a more elderly, heterogeneous group of hospitalised patients, prescribed GC. ${ }^{23}$

Machine learning techniques can be applied to clinical problems for risk prediction. We chose RF as it is a powerful ensemble machine learning method for predictive modelling, known to perform better than other methods smaller datasets. ${ }^{24}$ RFs work by constructing a large number of smaller decision trees and averaging the output of individual trees and are capable of capturing complex dependency patterns among multi-variate input features. As demonstrated by the heat maps (see online supplemental data), a large number of variables were collinear in their relationship and thus may be considered a potential cause for overfitting. RF outperforms other approaches in this respect and while it may mean causality may falsely be ascribed to a variable, it is known that collinearity does not affect the performance of RF as a predictor. ${ }^{25}$

The RF model accurately predicted the degree of hyperglycaemia for those without a history of DM in our population. Principal independent determinants were admission CRP, length of disease followed by thrombocytosis and anaemia, suggesting that systemic inflammatory burden and duration of IBD determined the risk of clinically significant hyperglycaemia in the cohort. No data of historic GC prescriptions were available so we cannot speculate further on whether the relationship with 
disease duration is a consequence of past GC exposure or a consequence of the disease process itself.

By using RF for early identification of those most at risk of significant GC-induced hyperglycaemia, alternative treatment strategies can be considered, including a rapid steroid taper, early use of biologicals and avoidance of oral steroids in those demonstrating adequate response to biological induction. Awareness of significant steroidinduced diabetes in this cohort appeared to influence physicians' decisions with an observed reduction in follow-on prescriptions for conventional steroid taper.

Although hyperglycaemia appears to resolve on stopping GC, this is not always the case. A large populationbased study associated previous oral GC with an increased incidence of future DM and in rheumatic diseases cumulative GC dose has been shown to be a risk factor for DM. ${ }^{26}{ }^{27}$ The IBD population, regardless of GC exposure, may be at higher risk of DM. A recent Danish populationwide cohort study suggests a higher incidence of type 1 diabetes among subjects with IBD and a Korean cohort study found IBD patients under 40 years to have an increased rate of incident diabetes diagnosis over 5 years, compared with age, sex and BMI-matched controls. ${ }^{28} 29$

This study has limitations. While we note that hyperglycaemia occurred during the admission of subjects treated with IVH, we do not know whether another aspect of the disease or its treatments was the principal cause. It is known that de-novo hyperglycaemia may also occur in hospitalised patients independently of GC as a result of the neurohormonal milieu of acute illness, with observational studies of critical care admissions reporting stress-induced hyperglycaemia in $17 \%-49 \% .^{30-34}$ As current guidelines recommend the use of high dose GC in moderate to severe acute IBD, there was no comparable cohort of patients managed without IVH treatment to try to better understand whether stress or GC was the predominant factor in our cohort. ${ }^{3}$ Despite an attempt to measure baseline HbA1c in all patients starting steroids, $29 \%$ did not have this checked, and 3\% had neither HbAlc nor CBG pre-IVH, so some cases of undiagnosed DM may have been missed.

Our model performed well as a predictive tool in our population, but we recognise that this was a prospective single centre study of fewer than 100 patient episodes and that its generalisability for hospitalised IBD in general remains to be established. Future research should aim to validate the model in a wider population and determine any effect of hyperglycaemia on clinical outcomes, which our study was not powered to achieve.

Intravenous GC have undoubtedly saved the lives of many IBD patients. However, there is significant associated morbidity, as acknowledged seven decades earlier by Truelove and Witts in their seminal work. ${ }^{1}$ It is questionable if GCs would be licensed in the regulatory framework currently applied to newer agents, given their side effect profile. In the ever-evolving IBD therapeutic landscape, it may be necessary to re-evaluate the position of GCs in the management of IBD. In UC, ciclosporin and infliximab are of equivalent efficacy as 'rescue' therapy for those demonstrating inadequate response to IVH; whether and for how long the preceding and concomitant GC course should be given for is not known..$^{35}$ The JAK inhibitor tofacitinib has demonstrated onset within 3 days in moderate to severe UC and has been used effectively alongside shorter IVH courses and budesonide as induction therapy in an acute severe UC case series. ${ }^{36} 37$

In acute $\mathrm{CD}$ the evidence for the benefit of GC to induce remission is less secure. ${ }^{4}$ Nutritional therapies can if given as exclusive enteral nutrition, be of similar efficacy as CD induction agents to oral GC. They may offer an alternative treatment strategy for those in whom steroids are contraindicated.$^{38}$ When coprescribed for a primary nutritional indication in our cohort, nutritional therapies did not increase the risk of hyperglycaemia.

Our data support the inclusion of CBG monitoring into standard clinical practice for IBD patients receiving IVH. Machine learning identified patients with a high inflammatory disease burden and a longer disease duration as being at the greatest risk of significant hyperglycaemia. Given the common complications of GCs and an enlarging landscape of biological, small molecule and nutritional therapies, there is an emergent case for researching steroid-free treatment strategies for acute IBD.

\section{Author affiliations}

${ }^{1}$ Department of Gastroenterology, University Hospital Southampton NHS Foundation Trust, Southampton, UK

${ }^{2}$ Human Health and Development, University of Southampton Faculty of Medicine, Southampton, UK

${ }^{3}$ NIHR Southampton Biomedical Research Centre, University Hospital Southampton NHS Foundation Trust, Southampton, UK

${ }^{4}$ Clinical Informatics Research Unit, University of Southampton Faculty of Medicine, Southampton, UK

${ }^{5}$ Department of Diabetes and Endocrinology, University Hospital Southampton NHS Foundation Trust, Southampton, UK

${ }^{6}$ NIHR Biomedical Research Facility, University of Southampton, Southampton, UK

Twitter Martin McDonnell @martinjmcd

Acknowledgements We would like to thank the diabetes multidisciplinary specialist team at UHS for helping to look after these patients.

Contributors MM, RJH, SD and MG were responsible for the original concept and planning of the study. MM, RJH, TM, SD, BZ and LD were responsible for clinical data collection and analysis. FB and HTTP were responsible for data extraction, analysis and modelling. RJH and MM contributed equally to this work and drafted the manuscript, which RF, TS, MP, JRFC, HTTP, MS and MG critically reviewed and revised.

Funding The authors have not declared a specific grant for this research from any funding agency in the public, commercial or not-for-profit sectors.

Competing interests MM: received non-financial support from Falk, MSD, Janssen and Takeda. RJH: personal fees from AbbVie and Janssen; non-financial support from Falk. LD: non-financial support from Janssen. SD: personal fees and non-financial support from Janssen; personal fees from Falk, MSD and AbbVie. JRFC: personal fees and research and/or educational support from Abbot, AbbVie, Amgen, Astra Zeneca, Biogen, Celltrion, GlaxoSmithKline, Janssen, Norgine, Pfizer, Pharmacosmos, Samsung, Shield Therapeutics, Shire, Takeda and Vifor. MG: personal fees from AbbVie, MSD and Takeda; non-financial support from AbbVie and Takeda

Patient consent for publication Not required. 
Ethics approval The protocol was reviewed and approved by the UK Health Research Authority - reference 19/HRA/0033.

Provenance and peer review Not commissioned; externally peer reviewed.

Data availability statement Anonymised data and random forrest code can be made available upon request.

Supplemental material This content has been supplied by the author(s). It has not been vetted by BMJ Publishing Group Limited (BMJ) and may not have been peer-reviewed. Any opinions or recommendations discussed are solely those of the author(s) and are not endorsed by BMJ. BMJ disclaims all liability and responsibility arising from any reliance placed on the content. Where the content includes any translated material, BMJ does not warrant the accuracy and reliability of the translations (including but not limited to local regulations, clinical guidelines, terminology, drug names and drug dosages), and is not responsible for any error and/or omissions arising from translation and adaptation or otherwise.

Open access This is an open access article distributed in accordance with the Creative Commons Attribution Non Commercial (CC BY-NC 4.0) license, which permits others to distribute, remix, adapt, build upon this work non-commercially, and license their derivative works on different terms, provided the original work is properly cited, appropriate credit is given, any changes made indicated, and the use is non-commercial. See: http://creativecommons.org/licenses/by-nc/4.0/.

\section{ORCID iDs}

Martin McDonnell http://orcid.org/0000-0002-7300-776X

J R Fraser Cummings http://orcid.org/0000-0002-9659-3247

\section{REFERENCES}

1 Truelove SC, Witts LJ. Cortisone in ulcerative colitis. BMJ 1955;2:1041-8.

2 Selinger CP, Parkes GC, Bassi A, et al. A multi-centre audit of excess steroid use in 1176 patients with inflammatory bowel disease. Aliment Pharmacol Ther 2017:46:964-73.

3 Gomollón F, Dignass A, Annese V, et al. 3rd European Evidencebased Consensus on the Diagnosis and Management of Crohn's Disease 2016: Part 1: Diagnosis and Medical Management. Crohn's Colitis 2017;11:3-25.

4 Ford AC, Bernstein CN, Khan KJ, et al. Glucocorticosteroid therapy in inflammatory bowel disease: systematic review and meta-analysis. Am J Gastroenterol 2011;106:590-9. quiz 600.

5 Hayashi R, Wada $\mathrm{H}$, Ito $\mathrm{K}$, et al. Effects of glucocorticoids on gene transcription. Eur J Pharmacol 2004;500:51-62.

6 Schäcke H, Döcke WD, Asadullah K. Mechanisms involved in the side effects of glucocorticoids. Pharmacol Ther 2002;96:23-43.

7 Waljee AK, Rogers MAM, Lin P, et al. Short term use of oral corticosteroids and related harms among adults in the United States: population based cohort study. BMJ 2017;357:j1415.

8 Conn JW, Fajans SS. Influence of adrenal cortical steroids on carbohydrate metabolism in man Metabolism 1956:5:114-27.

9 Umpierrez GE, Hellman R, Korytkowski MT, et al. Management of hyperglycemia in hospitalized patients in non-critical care setting: an endocrine Society clinical practice guideline. J Clin Endocrinol Metab 2012;97:16-38.

10 Braithwaite SS. Detection and management of diabetes mellitus during glucocorticoid therapy of nonendocrine disease. Endocrine replacement therapy in clinical practice. Humana Press, 2003: 254-5.

11 Weekes CE, Elia M, Emery PW. The development, validation and reliability of a nutrition screening tool based on the recommendations of the British association for parenteral and enteral nutrition (BAPEN). Clin Nutr 2004;23:1104-12.

12 International Expert Committee. International expert Committee report on the role of the $\mathrm{A} 1 \mathrm{c}$ assay in the diagnosis of diabetes. Diabetes Care 2009;32:1327-34.

13 World Health Organization. Definition, diagnosis and classification of diabetes mellitus and its complications: report of a WHO consultation. Part 1, diagnosis and classification of diabetes mellitus, 1999.
14 Breiman L. Random forests. Mach Learn 2001;45:5-32.

15 Pedregosa F, Varoquaux G, Gramfort A, et al. Scikit-learn: machine learning in python. J Mach Learn Res 2011;12:2825-30.

16 National Institite for Health and Care Excellence. Clinical guideline 32: nutrition support in Adults-Oral nutrition support, enteral tube feeding and parenteral nutrition. National Institite for Health and Care Excellence, 2006.

17 Thaiss CA, Levy M, Grosheva I, et al. Hyperglycemia drives intestinal barrier dysfunction and risk for enteric infection. Science 2018;359:1376-83

18 McGuckin MA, Eri R, Simms LA, et al. Intestinal barrier dysfunction in inflammatory bowel diseases. Inflamm Bowel Dis 2009;15:100-13.

19 Akerkar GA, Peppercorn MA, Hamel MB, et al. Corticosteroidassociated complications in elderly Crohn's disease patients. Am J Gastroenterol 1997:92:461-4.

$20 \mathrm{Ha} \mathrm{Y}$, Lee K-H, Jung S, et al. Glucocorticoid-induced diabetes mellitus in patients with systemic lupus erythematosus treated with high-dose glucocorticoid therapy. Lupus 2011;20:1027-34.

21 Panthakalam S, Bhatnagar D, Klimiuk P. The prevalence and management of hyperglycaemia in patients with rheumatoid arthritis on corticosteroid therapy. Scott Med J 2004;49:139-41.

22 Ito S, Ogishima H, Kondo Y, et al. Early diagnosis and treatment of steroid-induced diabetes mellitus in patients with rheumatoid arthritis and other connective tissue diseases. Mod Rheumatol 2014;24:52-9.

23 Donihi AC, Raval D, Saul M, et al. Prevalence and predictors of corticosteroid-related hyperglycemia in hospitalized patients. Endocr Pract 2006;12:358-62.

24 Floares A, Ferisgan M, Onita D, et al. The smallest sample size for the desired diagnosis accuracy. Int J Oncol Cancer Ther 2017:2:13-19.

25 Matsuki K, Kuperman V, Van Dyke JA. The random forests statistical technique: an examination of its value for the study of reading. Sci Stud Read 2016;20:20-33.

26 Gulliford MC, Charlton J, Latinovic R. Risk of diabetes associated with prescribed glucocorticoids in a large population. Diabetes Care 2006;29:2728-9.

27 Movahedi M, Beauchamp M-E, Abrahamowicz M, et al. Risk of incident diabetes mellitus associated with the dosage and duration of oral glucocorticoid therapy in patients with rheumatoid arthritis. Arthritis Rheumatol 2016:68:1089-98.

28 Halling ML, Kjeldsen J, Knudsen T, et al. Patients with inflammatory bowel disease have increased risk of autoimmune and inflammatory diseases. World J Gastroenterol 2017;23:6137-46.

29 Kang EA, Han K, Chun J, et al. Increased risk of diabetes in inflammatory bowel disease patients: a nationwide population-based study in Korea. J Clin Med 2019;8:343.

30 Dungan KM, Braithwaite SS, Preiser J-C. Stress hyperglycaemia. Lancet 2009;373:1798-807.

31 van den Berghe G, Wouters P, Weekers F, et al. Intensive insulin therapy in critically ill patients. N Engl J Med 2001;345:1359-67.

32 Plummer MP, Bellomo R, Cousins CE, et al. Dysglycaemia in the critically ill and the interaction of chronic and acute glycaemia with mortality. Intensive Care Med 2014;40:973-80.

33 Bersoux S, Cook CB, Kongable GL, et al. Benchmarking glycemic control in U.S. hospitals. Endocr Pract 2014;20:876-83.

34 Plummer MP, Finnis ME, Phillips LK, et al. Stress induced hyperglycemia and the subsequent risk of type 2 diabetes in survivors of critical illness. PLoS One 2016;11:e0165923.

35 Williams JG, Alam MF, Alrubaiy L, et al. Infliximab versus ciclosporin for steroid-resistant acute severe ulcerative colitis (construct): a mixed methods, open-label, pragmatic randomised trial. Lancet Gastroenterol Hepatol 2016;1:15-24.

36 Hanauer S, Panaccione R, Danese S, et al. Tofacitinib induction therapy reduces symptoms within 3 days for patients with ulcerative colitis. Clin Gastroenterol Hepatol 2019;17:139-47.

37 Berinstein JA, Steiner CA, Regal RE, et al. Efficacy of induction therapy with high-intensity tofacitinib in 4 patients with acute severe ulcerative colitis. Clin Gastroenterol Hepatol 2019;17:988-90.

38 Narula N, Dhillon A, Zhang D, et al. Enteral nutritional therapy for induction of remission in Crohn's disease. Cochrane Database Syst Rev 2018:4:CD000542. 